\title{
Making Sense of Complex Running Metrics Using a Modified Running Shoe
}

\author{
Paweł W. Woźniak \\ Utrecht University \\ Utrecht, the Netherlands \\ p.w.wozniak@uu.nl \\ Francisco Kiss \\ BETESO - Bürger Electronic GmbH \\ Waldenbuch, Germany
}

\author{
Monika Zbytniewska \\ ETH Zurich \\ Zurich, Switzerland \\ monika.zbytniewska@hest.ethz.ch
}

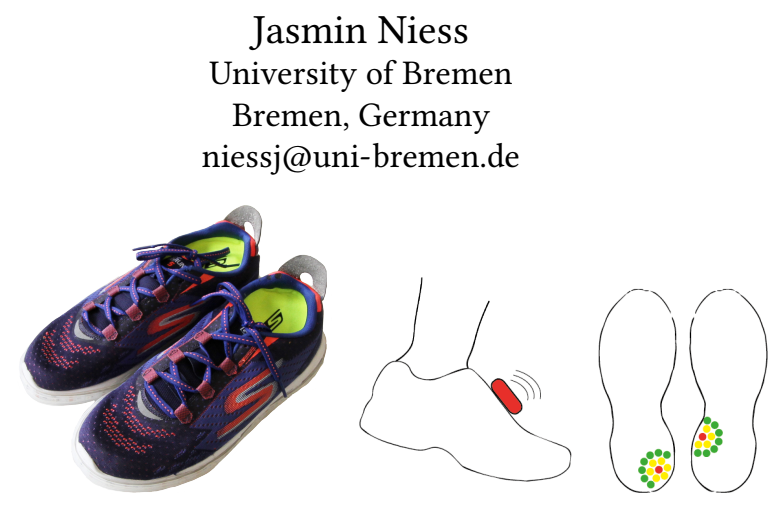

Figure 1: GraFeet is an additional pair of running shoes which uses data gathered by Runscribe sensors and visualises key foot mechanics metrics directly on the outsoles of two running shoes. When viewed from above, GraFeet looks like an ordinary pair of shoes.

\section{INTRODUCTION}

Government health programs and mainstream media try to persuade users to practise sports on a regular basis. Effectively, these campaigns lead to an increasing number of sport novices. Running is a popular sport for beginners because of its low entry threshold, among other reasons [14]. However, running comes at a cost of having to deal with fatigue, or even injuries. As the runner population increases, more and more people are at risk of running too much or committing running form mistakes, which may have severe health consequences [36].

Interactive technology is there to help runners be more aware about their bodies. New sensing technologies that quantify the way our bodies move when involved in sports are now easily available on the consumer market. As more data about how we run, swim or cycle becomes available and integrated in everyday devices, users have a growing interest in understanding the presented results. Further, they desire to relate the information to their bodies and reflect on their performance and wellbeing [41]. Current systems, e.g. Garmin connect ${ }^{1}$ usually present physiological data after the workout through dashboards that offer access to a multitude of measurement types, often visualised as time series graphs. While running apps used to display only GPS-based data (e.g. elevation profile and pace history), they now use extended sensing to display cadence, step length or heart rate based on wearable sensing. Despite the fact that users have access to an increased number of

\footnotetext{
${ }^{1}$ https://connect.garmin.com/
} 
metrics in commercial products, they still struggle to effectively reflect on their performance and desire to better understand their bodies [24, 41, 43]. A recent study has shown that while runners often know the factors that contribute to injury risk, they fail to 'listen to their bodies', relate their knowledge to their training and act accordingly, e.g. by training load reduction [38].

These facts suggest that current commercial solutions do not support reflection enough for users to know when to reduce or adjust training load based on sensor metrics. Additionally, as sensors become more complex, the amount of data presented increases and thus more effort is required from the users to gain insights. Consequently, a challenge for Human-Computer Interaction (HCI) is designing novel data presentation interfaces that enable amateur sportsmen to gain insights based on rich sensing data. These insights could then be used to better understand how training affects the users' bodies.

RunScribe $^{2}$ is an example of an enhanced sports sensing technology. A sensor is mounted on a running shoe to gather rich and accurate metrics about foot position and gait characteristics while running. These metrics can only be analysed after the run. As the data features information such as shock values and pronation (i.e. the side-to-side movement of the foot as one runs), users can identify how much strain they are putting on their legs. We propose moving beyond the dashboard to help users reflect on physiological data by visualising sensing data directly on sports equipment. In this paper, we discuss our design solution, GraFeet-a modified running shoe that relates sensor data to relevant areas of the foot (see Figure 1) for post-workout reflection. GraFeet empowers amateur runners to gain insight into their running form through gait metrics. We present findings from a user study where we compared GraFeet with the standard RunScribe desktop dashboard. We found that GraFeet was perceived as more usable and helped users generate more insights than the desktop system. We conclude with a set of research implications to inform the future design of sports-oriented physiological sensing.

This paper contributes the following: (1) the design and implementation of GraFeet-a modified running shoe for visualising physiological data; (2) a mixed-methods between-subject evaluation of GraFeet and (3) insights for designing future sport sensing systems that foster reflection.

\section{RELATED WORK}

In this section, we outline related work in the areas of HCI for sports and devices for data-driven reflection. We also describe how GraFeet fits into the space of reflection support systems for sports.

\subsection{Amateur Sportsmen and Technology}

A variety of research prototypes aimed at enhancing the experiences of runners has been developed in the field of HCI. Mueller et al. [31] looked at how running could be made a more social endeavor for distant runners by making them aware of their pace. In contrast, RUFUS [49] was developed to mediate the relationship between runners and their supporters during races by broadcasting position and allowing communication. In a similar vein, Mauriello et al. [26]

\footnotetext{
${ }^{2}$ http://runscribe.com
}

made running metrics publicly available by investigating how onbody displays can foster social interactions within running groups. Timmerman et al. [47] also aimed at supporting running groups by developing software for their organization. Strohmann et al. [44] studied on-foot sensors in the past, but focused solely on sensing accuracy and did not investigate the possible usage of the data. We noticed that while these systems looked at possible uses of running data, they did not attempt to provide reflection support for runners. Consequently, GraFeet explores how running metrics may foster reflection in runners.

A different strain of HCI work explores the role technology plays in the running experience and aims to understand the needs of runners with regard to technology. Tholander and Nylander [46] investigated the use of running watches and found that technology that enabled measuring performance and form was key for many athletes. Knaving et al. [24] explored the motivational aspects of running and found that qualitative post-run reflection was a desired activity for many runners and that they sought technological support for it. Similarly, JogChalking [5] was developed to help capture how runners felt during the run to enable them to log their affective state. These studies show that runners required further support for reflection to understand their running routines.

Past systems have also attempted to help users in improving form in sports. Runright [33] used real-time form feedback to foster better body balance. Similarly, Swing sound [34] produced auditory feedback to increase the bodily awareness of golfers. RunBuddy [17] looked specifically at how breathing patterns can be learned by runners. Hassan et al. [18] used an invasive approach where they used electrical muscle stimulation to correct foot movement of runners on a treadmill and Strohmann et al. [45] built a device for arm movement correction. All of these system examples used real-time feedback in hopes of the athlete changing their habits, but require a constant presence of the technology during practice. In our work, we explore an alternative solution; enhancing the users' understanding of their own bodies after the activity in order to help them improve their technique and avoid injuries. While we do not dismiss real-time approaches, we use lessons from past work on data-driven reflection, which primarily uses post-activity feedback, and apply it to running.

\subsection{Data-driven Reflection}

Our work is also inspired by recent trends in HCI that explore reflection as a desired way to improve oneself. Baumer introduced the notion [3] of reflective informatics: technologies that focus on ways an individual can reflect and foster change. Slovak [42] developed a framework which facilitated designing such technologies in a selfimprovement context. Saksono and Parker [37] showed that interactive artefacts could support story-driven reflection, demonstrating that recalling past sport-related events through storytelling may be highly beneficial. Our work explores whether data-supported reflection can be also used in the sports domain to understand one's body better and create conscious transformation.

Some technologies developed for athletes hint at enabling reflection. TickTockRun [23] is an information visualisation tool embedded in a clock aimed at triggering family discussions about performance metrics. Sweat Atoms [22] use running metrics to 
3D-print small tangible artefacts to portray the athlete's activity level. McGookin and Brewster [28] postulated building tools that enabled runners to spontaneously make decisions on which locations to choose for activities. Wozniak et al. [50] studied data sharing for multiple sports and found that collecting and reflecting upon qualitative data was an important part of outdoor activities for some users. Recently, Sauvé et al. [40] developed a situated tangible artefact that visualised fitness tracking at home. They argued that tangible objects were more likely to trigger reflection thanks to their visual appeal. These systems illustrate the potential benefits of reflection in the physical activity domain. GraFeet goes beyond past work by offering the first (to our knowledge) systematic evaluation of an artefact designed for reflection in sports. Further, we focus on bodily awareness to investigate if users can benefit from running metrics to better understand their running technique.

A recent strain of work in HCI has advocated designing artefacts for reflection as an alternative to supporting behavioural change. Brynjarsdottir et al. [9] advocated abandoning persuasive approaches and designing for reflection in the sustainability area. Baumer [3] postulated building tools that empower reflection in the area of personal informatics. Inspired by these works, we aimed to design an artefact that can serve as a vehicle for analysis and reflection. As a review of past work on reflection in HCI [4] indicated that reflection and self-knowledge often go in pairs, we endeavour to investigate whether this can be applied in the sports domain.

\subsection{Current Approaches to Using Data for Reflection in Running}

While many systems designed to support the runner collect large amounts and different types of data, the presentation of such data has not been extensively explored. Commercial products focus on time series graphs. Running pods (Stryd ${ }^{3}$, Garmin Pod), chest straps $\left(\right.$ Wahoo Tickr ${ }^{4}$ ) or socks $\left(\right.$ Sensoria $\left.{ }^{5}\right)$ rely primarily on line charts with Sensoria only recently offering a static foot drawing next to the graph. Commercial smartphone applications for running (e.g. Runkeeper ${ }^{6}$ or Strava ${ }^{7}$ ) offer extensive dashboards for reviewing data. As injury rates for runners are not dropping [36] while running apps are widely used [19], it appears that current commercial solutions offer little support for reflection. Further, current apps put speed and location in focus but not how the body of the runner works and feels.

Understanding one's run has a high potential for improving the running experience. Meaningfully reflecting on one's personal informatics data has been established as a pursuit that can benefit personal wellbeing $[3,13]$. Previous work, e.g. [18], showed that interactive systems can help change running technique and improved technique can reduce injury risk [30]. Further, multiple works have found that physically active individuals desire more information about their activity, e.g. [24, 25, 32] and require data-driven social support [12]. Another known issue is that those who are serious about running often fail to take a step back and listen to their bodies [35]. Consequently, not taking enough time to rest when required

\footnotetext{
${ }^{3}$ https://www.stryd.com/

${ }^{4}$ https://eu.wahoofitness.com/devices/heart-rate-monitors/tickr/buy

${ }^{5} \mathrm{https}: / /$ www.sensoriafitness.com/

${ }^{6} \mathrm{http}: / /$ runkeeper.com

${ }^{7}$ https://www.strava.com
}

is often the reason for injuries. Thus, we decided to design a device that would facilitate reviewing one's bodily measurements after a run and prompt post-run reflection. The aforementioned works also reported that current technologies for physical activity could offer better support for data-driven reflection.

Our work explores alternative ways to present running metrics in order to foster a better understanding of one's run. Previous HCI work showed that situated tangibles can effectively trigger reflection $[22,40]$. GraFeet uses a shoe-based display in anticipation of future possibilities of sole augmentation [11]. Our system is designed for advanced amateur runners [24] who are curious about their gait properties.

\section{DESIGN}

GraFeet is a proof-of-concept prototype which aims to provide a generative contribution to designing reflection systems for physical activity. We initiated our design process motivated by the fact that running literature often stresses the importance of post-run reflection.

As we wanted to look for alternatives to current solutions, we decided to design a solution that would not use a dashboard but would incorporate extended bodily sensing. As lower extremities are the key injury area for runners, we focused on foot sensors and used an advanced commercial foot sensor, RunScribe. Further, inspired by Lumahelm [48], we were drawn to the relatedness and ease of understanding that augmenting sports equipment produced in users. Additionally, we noted that using tangible objects was also identified as beneficial for designing health reflection technologies [1].

Consequently, we decided to augment the most important piece of runner's attire - the running shoe. We opted against a screenbased visualisation solution as we wanted our artefact to be easily relatable to one's body and past work has shown that fostering reflection with traditional desktop- and mobile-based solutions may be problematic and eventually lead to lapses in usage [13]. We also aimed at designing a prototype that would be embedded easily in a home environment (much like LOOP [40] or TickTockRun [23]). Conceptually, we aimed to subtly prompt reflection in users rather than promote change as our target user group consisted primarily of physically active individuals. Further, we believed that presenting the data in the form of a shoe, an artefact easily associated with the running experience, would help develop a more personal relationship with the data. We imagined a system that would enable runners to reflect on even minute changes in their physiology and aid in training planning and, if used long term offer the potential of preventing injuries.

As the Runscribe sensor provides multiple metrics, visualising all of these would not only confuse the user (as preventing data clutter is a key consideration when designing dashboards [15]), but also require them to have the kinesiology knowledge needed to interpret the data. As a consequence, we consulted reference books [10] and experts in kinesiology (two senior researchers from the local sports science department) to determine key metrics that facilitate understanding of one's running on a foot level in order to prevent injuries. These metrics were: foot strike type (which part of the foot, front or back hits the ground first while running), 
pronation (the lateral bend of the foot) and impact Gs (the vertical force with which the runners hits the ground every step).

Consequently, the initial design requirements called for a system that:

- offered a simple, glanceable visualisation;

- would convey the key metrics (foot strike type, pronation and impact Gs) in one view;

- take advantage of the familiarity of a tangible artefact that could be situated with a home;

- used a form strongly associated with running;

- would be suited for a post-run reflection scenario.

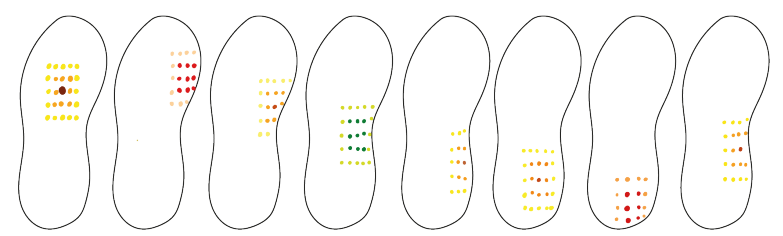

(a) The visualisation used in the final prototype. Foot strike type, pronation and shock are visualised on the outsole. The location of the most intensive point shows foot strike type ( $\mathrm{Y}$ axis in the picture) and the value of pronation ( $\mathrm{X}$ axis in the picture). The colours map shock to community average shock with red representing relatively high shock, yellow showing medium shock and green showing low shock.

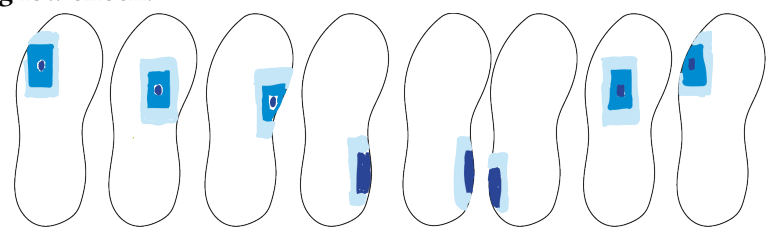

(b) Alternative colour version of $2 \mathrm{a}$

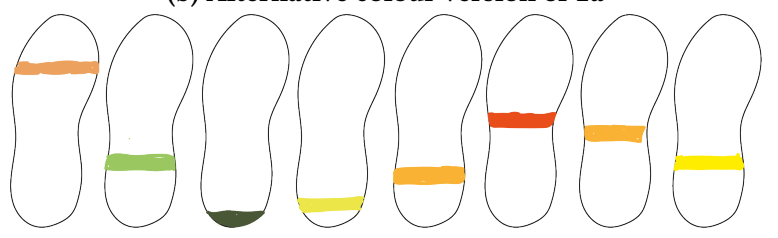

(c) Alternative visualisation: foot strike type and impact Gs are presented on the shoe sole and pronation is shown on the side of the shoe. The colour mapping relates the shock to community average shock. Location of the colourful stripe corresponds to foot strike type.

Figure 2: Three digitisations of paper prototypes for visualisations to be used in GraFeet. The protoypes were used in our pre-study to elicit feedback about how a shoe-based visualisation may help understand running metrics and choose which visualisation to implement.

\subsection{Designing the Visualisation}

Having established the requirements, we investigated how we could design a visualisation that would prompt users to reflect and provide key metrics meaningful for their running technique.

To that end, we built a series of paper prototypes to investigate colour patterns and shapes for visualising the metrics. Further, we used a modelling clay shoe for users to explore different locations

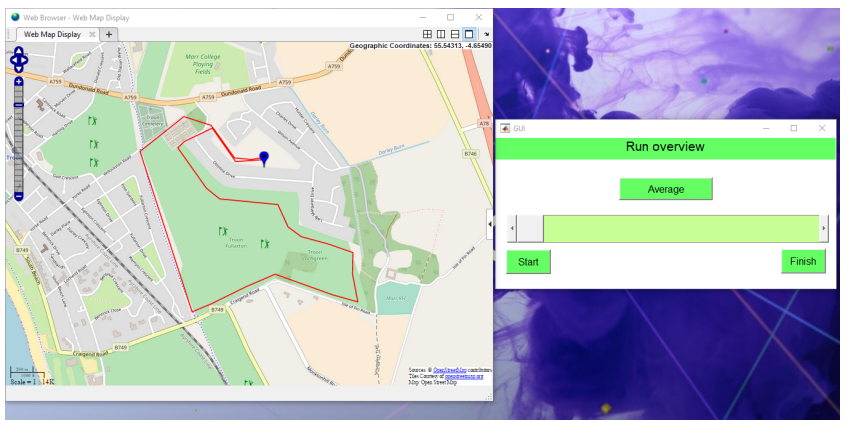

Figure 3: The desktop interface accompanying GraFeet. The user can view the run on a map and navigate to different parts of the run using the slider. The appropriate metrics are then shown on GraFeet. Average foot metric values can be shown by pressing the button. Thus, the interface provides both instantaneous metrics and an overview of the gathered data.

on the shoe for visualising data. We conducted a design pre-study with 17 participants who all reported having been active runners (11 males and 6 females, aged 23-46). We explained the metrics to the participants and then discussed their importance for running technique and avoiding injuries. We then used the clay shoe and paper prototypes to look for possible visualisations. We also asked them to draw paper prototypes of possible visualisations. Users considered visualising data on the side of the upper of the shoe, on the outsole or on the entire surface. We observed that participants initially struggled to connect the metrics to running technique or areas on their feet. The majority (14 participants) preferred visualisation only on the bottom of the shoe. Consequently, we presented users with alternatives of how data could be shown on the outsole. Figure 2 presents the three most popular alternatives. After iterative refining, we found a solution that appealed to most users and conveyed the three target metrics shown (Figure 2a). Users preferred a visualisation that used a green-to-red mapping for shock values. We used the community averages provided by Runscribe to adjust the mapping, i.e. the average shock value was mapped to yellow.

\subsection{Final Prototype}

The final prototype, which we named GraFeet (Graphical representation on Feet), consists of two running shoes in which outsoles were replaced with LED matrices for visualisation. Thus, GraFeet offers a tangible interface for visualising foot sensing data. The user first affixes the Runscribe sensors to his/her running shoes (the sensor can be mounted on the back of the shoe or on the laces) and completes a run. Afterwards, the data is transferred to GraFeet for analysis. We built a simple desktop interface that visualised the run on a map and enabled the user to choose a particular moment of the run using a slider (shown in Figure 3). The state of the foot metrics would then be shown on GraFeet. In pilot studies during the design process, we observed that that some users' data showed significant differences between the left and right foot. Thus, we opted for building two shoes to avoid any ambiguity. The final version of GraFeet is shown in Figure 4. 


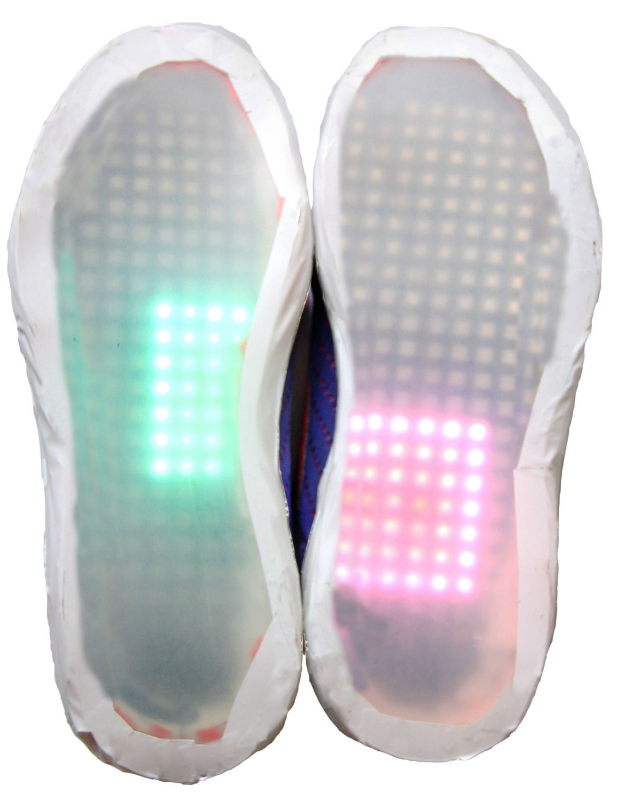

Figure 4: The final version of the GraFeet visualisation, presented on two running shoe outsoles. The right shoe (left) shows a mid-foot strike, pronation towards the inside of the foot and light shock. The left shoe (right) shows a strike close to the heel, inside pronation and high shock.

\section{IMPLEMENTATION}

GraFeet uses Sketchers GoRun 5 running shoes. We removed most of the outsoles of the shoes and replaced them with Flexible 8x32 NeoPixel RGB LED Matrices covered by a layer of $80 \%$ transparent silicone. The matrices are driven by an Arduino UNO connected over USB to a laptop. We used the two-shoe Runscribe sensor for our prototype. This device version has been shown to offer rich and accurate running metrics, which was validated in multiple studies that used motion tacking ${ }^{8}$. We chose Runscribe as the technology of choice as it offers the largest number of metrics and it is the first widely available running gait sensor.

Sensor data from Runscribe is transferred via Bluetooth to an Android Smartphone and then uploaded to the Runscribe dashboard. It is worth noting that the version of Runscribe used in this work does not provide real-time feedback. The data is then exported from Runscribe as a CSV file. Additionally, we use Runkeeper to gather GPS data and export it as a GPX file. The CVS and GPX files are then imported in Matlab for processing, providing the desktop interface and calculating the visualisation to be shown on the shoe. Having implemented the prototype, we conducted a user study to evaluate it.

\section{EVALUATION}

We conducted a user study to evaluate GraFeet and compare it with the baseline Runscribe interface (a web-based dashboard for

\footnotetext{
${ }^{8}$ https://runscribe.com/tag/validation/
}

post-run data, presented in Figure 5). We decided to compare GraFeet with the standard Runscribe solution as we endeavoured to investigate how much of a possible improvement our system offered over a solution available on the consumer market. An alternative that we considered and rejected was developing a desktop-based version of the visualisation as displayed on GraFeet. We decided to not compare two custom-made artefacts to understand more about our design and compare it to a state-of-the art runner gait analysis tool [20]. However, in order to use a real-world baseline, we decided that we would use a data source that offered more metrics than GraFeet. The dashboard offered additional information such as: symmetry, step rate, contact time and flight ratio ${ }^{9}$.

Thus, we investigated how GraFeet differed from a traditional desktop-based solution. We designed a mixed-methods betweensubjects experiment where users gathered foot sensor data during a short run. They would then analyse the gathered data in one of the conditions. While we considered a Wizard-of-Oz approach with users analysing predefined data, we opted for increasing the ecological validity of the study by looking at data directly after a run. This enabled us to gain deeper insight into the respective technology's potential for reflection. We used the following alternative hypotheses for the study:

H1: GraFeet would be significantly more usable than the baseline dashboard. We expected that using GraFeet would be perceived as more usable by users due to its reduced complexity compared to the dashboard.

H2: GraFeet would produce significantly more data-driven insights than the dashboard, despite the more limited range of data offered. In line with past work, we expected that an innovative, tailor-made, tangible interface would produce more insights than a traditional desktop dashboard [21].

The study used one independent variable; type of data visualisation interface. The interfaces used were GraFeET and DAshboARD.

\subsection{Data Collection}

We gathered quantitative and qualitative feedback from the participants to understand their experience of using GraFeet.

5.1.1 Measures. The following dependent variables were measured for both conditions:

Usability and user satisfaction index [0-100 points] measured using the System Usability Scale (SUS) [8]. We administered the standard SUS questionnaire to measure the perceived usability of GraFeet and compare it to the baseline system.

Number of insights and 'anti-insights' [insight count]. We used the insight reporting methodology [39] to assess how much insight on one's running technique the user was able to produce based on the data. We employed this metric to gain an assessment of the reflection potential of the two solutions. The users were presented with the insight questionnaire with the question 'What have you learnt about your running technique?' Additionally, we audio recorded while the participant was analysing the data. The insight/anti-insight count was obtained by closed-book coding the data in the questionnaire answers and the recording. We adopted this methodology as we were looking for very specific information

\footnotetext{
${ }^{9}$ Sample dashboards can be viewed at https://dashboard.runscribe.com/
} 


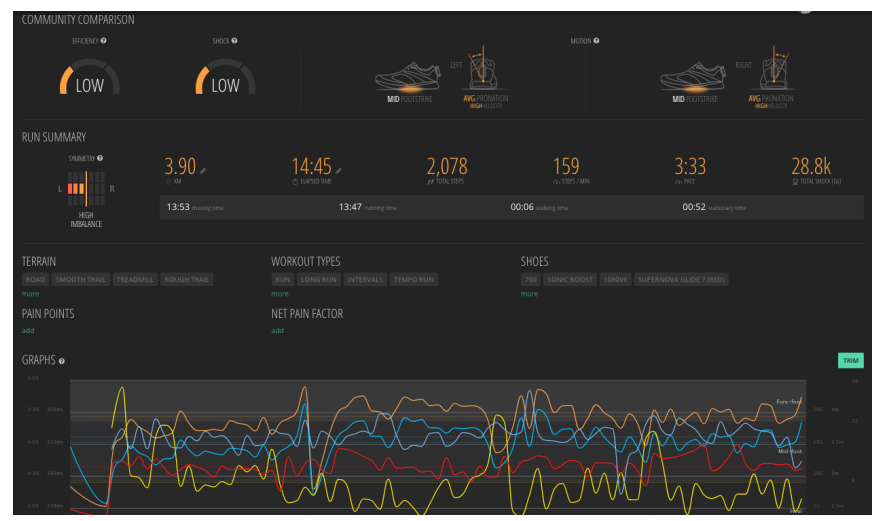

Figure 5: The standard Runscribe web dashboard. Users are presented with an overview of the run using average data (top). They can also choose metrics to plot and review in time (bottom).

in the data and report quantitative metrics as suggested by Blandford et al. [6, p.68]. We defined an insight as any connection the user made between the data and their body mechanics, e.g. 'This indicates I land on my toes when running downhill.' We counted any expression of confusion or request for additional knowledge as an anti-insight, e.g. 'I have no idea what this number means.'. Two researchers independently coded the notes produced by the participants with high agreement (Cohen's $\kappa=.93$ ).

Perceived percentage of insights learned [\%]. In line with the method proposed by Saraiya et al. [39] we also asked participants for their subjective assessment of how much they had learnt from the data. A scale of 0-100 percent was presented to users along with the question 'How much of the total potential insight do you feel you have obtained?'

5.1.2 Interview Protocol. We also collected qualitative data by conducting a semi-structured debriefing interview. In the interview we asked participants about their perceived understanding of the data and how they related the presented values to their body mechanics and running technique. We also inquired whether they recalled specific moments of the run and if those moments were reflected in the gathered data. Finally, we were interested about envisioned benefits from bodily sensing while running and the information the users would like to have about their own bodies related to running. The interviews were transcribed verbatim and relevant passages were selected by two independent coders (full agreement was reached) We then used affinity diagramming to identify recurring themes relevant to our work in iterative discussions.

\subsection{Participants}

We recruited $N=36$ participants (10 female, 26 male, aged 18-32, $M=26.00, S D=2.73$ ) through our university mailing list and by distributing posters around the campus. Participants attended the study individually and did not communicate with other participants. We required that users declare they were able to run for $20 \mathrm{~min}$ utes and arrive in their own running equipment. All participants declared to be advanced amateur runners, training more than once

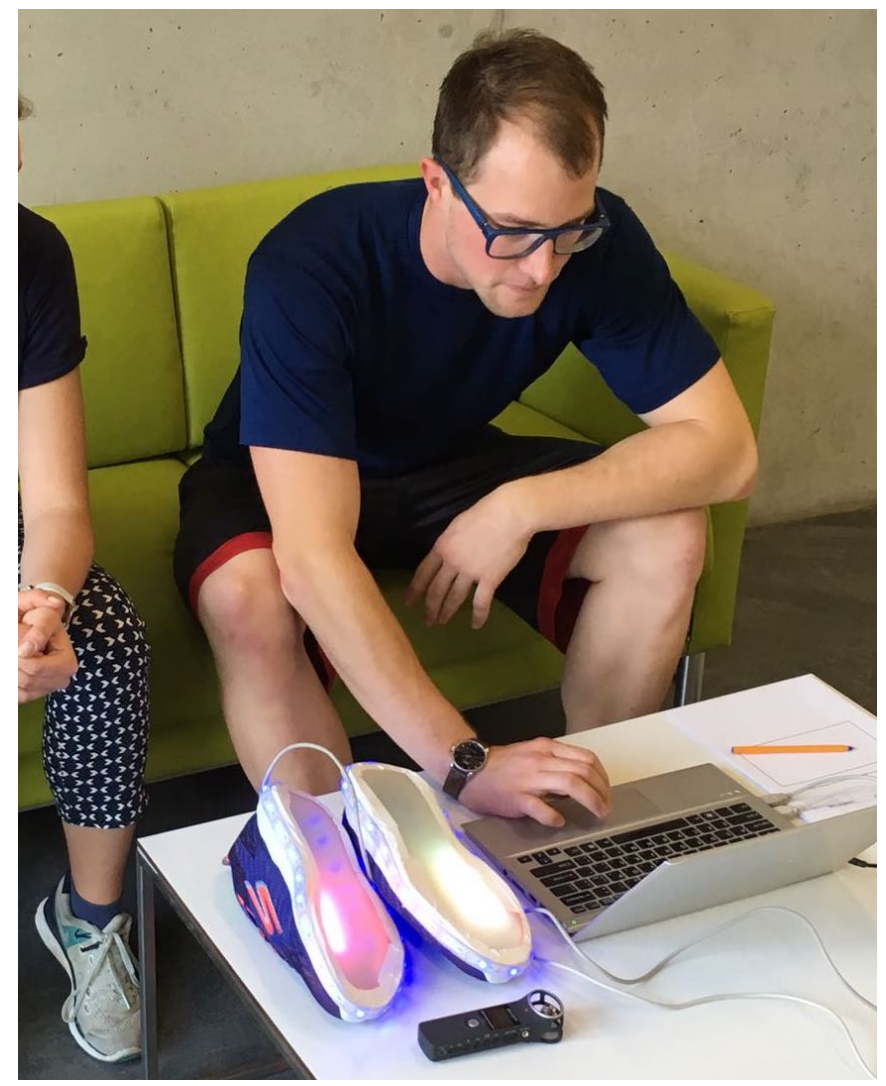

Figure 6: A runner analysing foot sensor data with GraFeet. Users could manipulate the GUI on the laptop to choose a particular time for which data was shown on GraFeet. They were encouraged to think out loud and the entire process was audio recorded.

a week and committed to a running regime (i.e. they all belonged to the user group defined by Knaving et al. [24]). There were no participants with professional running experience. Each participant was remunerated with EUR 10. Additionally, isotonic drink and water were available throughout the study.

\subsection{Apparatus}

We used Runscribe version 2 (consisting of two pods) for data gathering. We affixed the Runscribe sensors to the users' shoes according to their preference, in the lace or heel area of the shoe. The data was then uploaded to the Runscribe system using a Nexus Phone. In the GraFeEt condition, the data was visualised on two GraFeet shoes connected to a Sony Vaio Laptop (13" screen diagonal). The same laptop was used to display the Runscribe dashboard in the Google Chrome browser in the Dashboard condition. The study took place in a university building adjacent to a forest with running paths.

\subsection{Procedure}

Having welcomed the participants to the study, we asked them to complete a consent form informing them about all the data 
collected. The participant was then assigned an experimental condition (GraFeEt or DASHBoARD). Conditions were alternated between the participants. This limited the possibility of them exchanging information when leaving or entering the research facility. After verifying that a user was ready to run, we explained the purpose of the study and asked where to mount the Runscribe sensors on the user's shoes. We then informed the user that the sensors recorded foot motion data and they would be later asked to review and reflect on the information gathered. Once we affixed the sensors and verified they were operational, the user was instructed to run a signposted route of about $1.75 \mathrm{mi}$ from the study location and back. While we provided a suggested route, the participants were free to explore the area and run longer, until they felt they were ready to look at the data. They were instructed to keep a comfortable pace throughout the run in order to prevent exhaustion affecting their perception of the interface used. After a runner returned from the route, we let him/her rest and drink if desired. In the meantime, we transferred data from the Runscribe pods to the phone. Next, we presented the user with the interface assigned to him/her (GraFEET or DASHBOARD) along with the insight questionnaire and instruction to reflect on the data to complete the task. No time limit was specified and the users were instructed to generate as many insights as possible and think out loud. The experimenter was available to answer any questions and the entire analysis was audio recorded. Figure 6 shows a participant engaged in analysis using GraFeet. Once done, we administered the remaining questionnaires and conducted the semi-structured debriefing interview.

\section{RESULTS}

The participants completed 36 runs at an average time of $M=$ $16.35 \mathrm{~min}(S D=6.14 \mathrm{~min})$ and a distance of $M=2.34 \mathrm{mi}(S D=$ $1.31 \mathrm{mi}$ ). One participant completed the run early due to sudden rain.

\subsection{Usability (SUS)}

The grand mean of the usability score was $M=71.53(S D=12.64)$. GraFeET was perceived as more usable with $M=76.39$ and DASHBOARD was rated on average $M=66.67$. A Mann-Whitney $\mathrm{U}$ test revealed a significant difference $(U=94, p<0.05)$.

\subsection{Perceived Insights Learned}

The participants reported an average of $M=61.11 \%(S D=18.94 \%)$ perceived insights. DASHBOARD produced more perceived insights with $M=63.89 \%$. GRAFEET produced $M=58.33 \%$ perceived insight. A Two Sample t-test revealed no significant difference between the conditions $(p>0.05)$. Below, we present two typical insights:

I learned that my right and left feet vary in strike type. $(P 6, G)$

Here is what happened to my feet when I stopped to tie my shoe laces. (P11, D)

\subsection{Insights and anti-insights}

The average combined number of insights (gathered from the insight questionnaire and the think-aloud recording) was $M=6.25$ $(S D=2.94)$. Using GraFeEt led to more insights with $M=7.89$, while DASHBOARD generated an average of $M=4.61$. We conducted

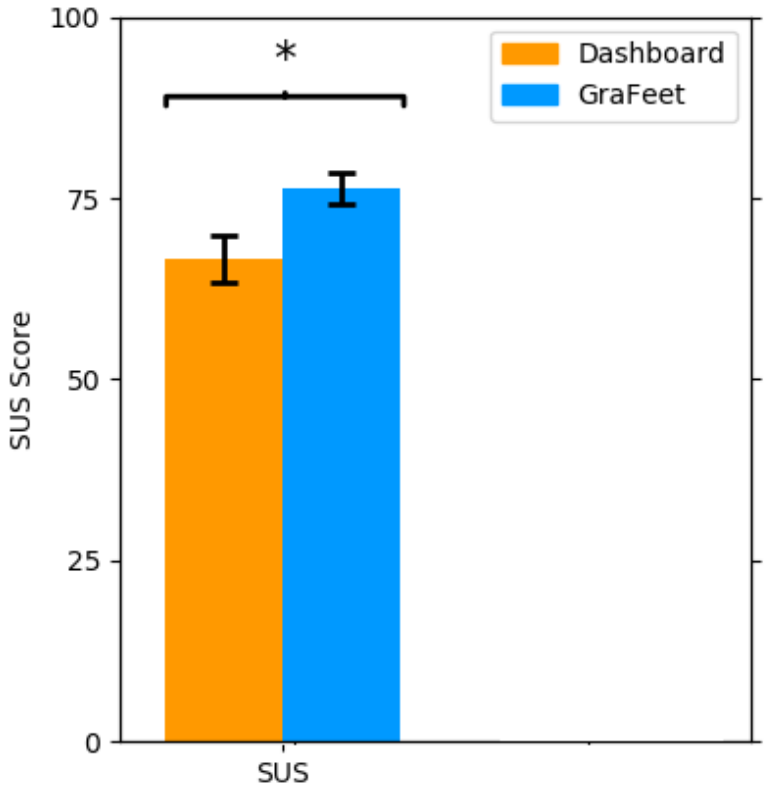

Figure 7: Average SUS scores for Dashboard and GraFeet. Error bars show standard error.

a Two Sample t-test to determine that there was a significant difference between the conditions $(t(34)=3.99, p<0.001)$.

The grand mean of anti-insights generated by the participants was $M=2.14(S D=2.18)$. A Two Sample t-test showed that DASHBOARD $(M=3.22)$ produced significantly more anti-insights than GraFeet $(t(34)=3.40, p<0.01, M=1.06)$. The examples below represent what was coded as an anti-insight:

What does efficiency mean in this context? $(35, D)$

It looks like this should say more about how elevation affects pressure. $(36, G)$

\subsection{Interviews}

The participants provided extensive feedback about their data analysis process. Here, we present a selection of the data relevant to the evaluation of GraFeet. We present excerpts from the interviews and comments recorded during the study accompanied by the participant number and the interface they used (Dashboard or GraFeet) in order to highlight the findings that stem from the differences in the interfaces. Using affinity diagramming, we classified the interview data into three categories (form factor, contextualizing data, potential for reflection).

6.4.1 Form Factor. Some of the users commented specifically on the idea of presenting the data on an object rather than a traditional screen. They found that it empowered them to have an overview of the situation at a given time point during the run and made the data more relevant: 


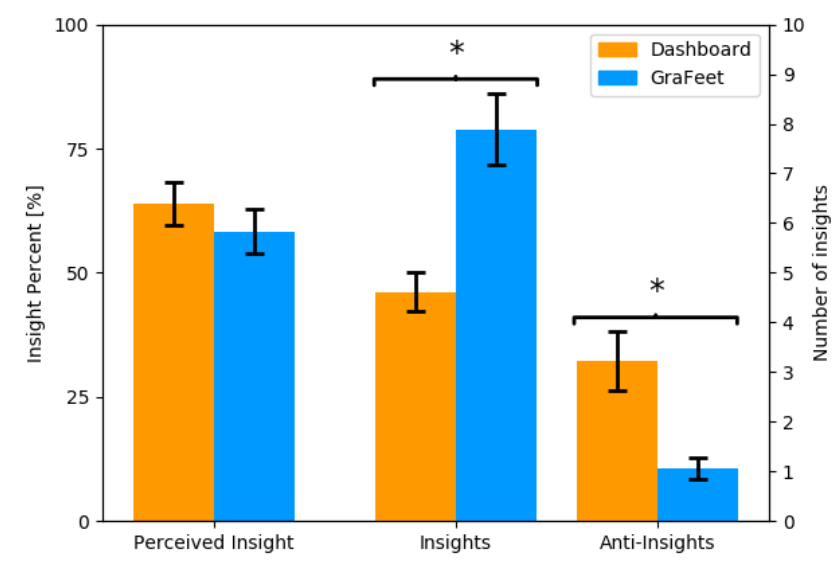

Figure 8: Average perceived insights in percent, the count of insights and anti-insights for Dashboard and GraFeet. Error bars show standard error.

I like the physical object showing me the data. It makes more impact. You can actually see and realize what is happening exactly and it gives you a more real feeling about the data. $(P 15, G)$

Furthermore, participants commented on how GraFeet used a shoe to represent gait data. They found it useful to relate the position of the visualisation to the location on their feet. P5 commented:

I like the colours, you see directly how much pressure it is, you see red or yellow, you see where it is. $(P 5, G)$

In contrast, the participants considered the dashboard interface to be very professional. One participant remarked that the software had a scientific look and thus it boosted their confidence in having learned about their body:

I learned a lot about my body, it looks neat, appealing, scientific. There are a lot of terms which a normal person wouldn't know, but if they just google it they'll learn. It looks scientific, so it gives you scientific data about you. $(P 1, D)$

6.4.2 Contextualising Data. Many interview answers addressed the process of putting gait and foot data into context. Firstly, GraFeet triggered curiosity about one's running mechanics:

It was interesting which part of my feet is responsible for my running. $(P 24, G)$

We have also observed that runners often looked for the means to make data actionable. Once they were convinced that they understood the data correctly, they wanted to know how they could react to improve their technique:

I would like to get some information about what I should change to make my running experience better. More like a comparison sort of thing. More guidance. $(P 11, D)$

In contrast, P13 was able to connect data from GraFeet with an ongoing injury and understand how it affected their technique:
I could see that my knee injury is affecting my running. That I'm running with the inner side, that's something I didn't know. $(P 13, G)$

Some of the users explicitly requested that baseline information should be provided. While a running style is a very unique personal feature and different running styles work well for different people, users felt the need to know an ideal style:

It would be good to know a reference - what is the optimal running style. $(P 25, G)$

The need for a baseline was often connected with a more explicit need for the necessary kinesiology knowledge to be available at hand. Users recognised that many of the metrics in the Dashboard were quite advanced and an explanation should be provided:

The terms I didn't understand are those that no one who's not in this field would be able to understand. Sport scientists will know it, but a person who's not in this field would not get it. (P35,D)

They also wondered whether the observations they made were positive or negative:

I learned that my left and right foot are different, but I don't know what to do about it, how to change it. (P32,D)

Many participants were confused by the abundance of data offered in the dashboard. They not only required reference metrics, but also wondered about the meaningfulness of the metrics. One participant requested embedding knowledge in the interface:

I don't know what an average [pronation] is, I can't really compare with the data. I don't know, for example, what a good number of steps is for an average runner. Without this knowledge I can't really understand the visualization. I liked the graph. The shock, efficiency metrics, I would probably need to have a background to analyze it. $(P 11, D)$

6.4.3 Potential for Reflection. Finally, we noted that participants often considered how body-worn sensors could help them to reflect after completing a run. Another runner described how they got lost trying to run the prescribed study route:

I could remember a point in the forest where there was no sign any more, then I got attacked by a dog, then there was no sign any more so I just stopped, then I just ran in circles, because I was confused by the way again. (P29,D)

Users connected their qualitative experience of the run with the data which enabled them to recall qualitative details about the workout:

I learned about which part of the foot was used during the run (...) and how much relaxed I was during running and when I paused. What I knew before is that when running in the forest, when the road is not so smooth, and it's more difficult to run, you use the inside of your foot. $(P 17, G)$

Some of the users reported an increased sense of understanding of their body after using GraFeet. One participant remarked that they 
could estimate the way different terrain would affect their body based on the insights from the data:

The visualization gives you a complete idea about which feet was used and relaxed the most at which point of the run. It also gives you an idea if there are differences in the terrain, how would that affect your body. $(P 33, G)$

Finally, the majority of GraFeet users expressed a wish to use it longer and anticipated that interfacing with foot sensor data could lead to a better bodily awareness and improved running technique:

This is giving me insight into my body, but if I used it regularly I could adjust myself a bit more. To make sure that my metrics are same (balanced) and I would try to improve my speed. $(P 36, G)$

\section{DISCUSSION}

In this section, we first attempt to understand the differences in how users interacted with the Runscribe dashboard and GraFeet. We then draw on this explanation to showcase insights for future post-activity data reflection interfaces for sports.

First, we must note that given the different functionalities that the solutions provide, we did not aim to determine the preference between the two systems. For that reason, we employed a betweensubject design. Our study found that GraFeet was perceived as significantly more usable than the desktop interface. Thus, H1 was confirmed. Yet, both systems scored as 'good' on the SUS according to Bangor et al. [2].

We hypothesise that the Runscribe dashboard features is a professional dashboard [15] aimed primarily at users with a deep knowledge of foot mechanics and its potential for sports reflection is limited. In our study, the dashboard produced more anti-insights, which shows that the users found it hard to relate the data presented to their running. In contrast, GraFeet offered a limited amount of metrics in a form that reminded users of the activity and enabled them to relate the data points to their bodies. Our interview data shows that although users were consciously looking in the dashboard for knowledge about their own body, they were unable to obtain it without prior extensive knowledge in body mechanics. Consequently, future systems should reduce the number of metrics shown or empower the user to filter early. With the ever-increasing number of sensors, presenting too many details can eventually lead to cognitive overload [27]. However, we also see a need for extensive metrics to be available if a user endeavours to develop an expert understanding of the data at a later stage. Our work suggests that advanced data solutions for running can only produce a positive user experience if presented on request to users with extensive theoretical knowledge of kinesiology.

We observed that GraFeet empowered users to generate significantly more insights about their foot mechanics than the dashboard. Consequently, we consider $\mathbf{H} \mathbf{2}$ confirmed. The users were able to effectively reflect in a post-workout scenario. This hints that GraFeet was able to benefit from the immediate qualitative experience of physical activity, which enabled users to contextualise data better. This is in line with past work, which indicated that users often consider qualitative aspects of exercise as important despite the fact that traditional sports applications focus on numbers $[28,50]$. As a consequence, the time period immediately following a finished exercise session appears to be specifically suited for reflection. This is an unexplored design space as current approaches either aim to provide feedback during the exercise (e.g. $[18,33]$ ) or use artefacts promoting reflection constantly throughout the day [40]. GraFeet shows that future design should use the time immediately following the workout as an opportunity for reflection in order to enable users to connect their qualitative experience of the activity to the data presented and enable data-supported skill improvement. As users are known to be particularly energetic in the post-workout period (i.e. experiencing the 'runner's high' [7]), it can even be used to foster data-driven experimentation, which has been shown to be beneficial to wellbeing [29]. This, in turn, can be used to design for long-term exposure to data, which was shown to be a key factor for reflection [3].

The qualitative data gathered in our study shows the importance of reference values when designing for interacting with sensor data for sports. While the most commonly tracked metrics such as distance or pace do not require explanations for the user, we observed how the more advanced foot parameters required a frame of reference. GraFeet emphasised how the user's sensor data related to community averages thus enabling users to operationalise raw numbers (e.g. in terms of footstrike shock values). Thus, we see that future systems for sports that use body-worn sensors should provide reference metrics in a clear manner. This way, interfaces can help the user make quantitative metrics meaningful and translate them to qualitative descriptions of how one's body functions.

An interesting discrepancy in our study is that while we noted that users gathered more insights using GraFeet and they were more confused when using the Dashboard, there was no significant difference in the reported insight percentage. Thus, we cannot conclude which system made users feel they gained more knowledge. We hypothesise that this apparent inconsistency can be attributed to the fact that the dashboard had a professional and, to a degree, intimidating design, which could in turn produce social desirability bias [16]. An abundance of data points created a perception of being able to access extensive information. On the other hand, sensemaking using this extensive information could only be successful with extensive background knowledge that the users did not possess. In contrast, parts of the needed knowledge were embedded in the design of GraFeet. For example, changes in pronation were reflected by lateral movement of the visualisation on the outsole of the shoe thus showing the meaning of pronation without explicitly naming the metric. This hints that GraFeet, as a tangible visualisation artefact, may have benefited from some of the qualities usually attributed to data physicalisation [21]. We see a need for sports sensing interfaces to communicate the background knowledge necessary to understand sensing metrics and couple it closely to the visualisation. This was apparent in the dashboard interface. While textual descriptions of the metrics were available, none of the participants used them.

\subsection{Limitations and Future Work}

While we believe that our work offers comprehensive insights into designing post-workout reflection experiences for runners, we do recognise that our research is prone to certain limitations. Firstly, we 
recognise that we only looked at a single instance of post-exercise reflection per user. Future studies can take a more holistic look at the post-workout experience by conducting studies where runners use sensors over a longer period of time. This could also enable investigating whether reflection can lead to eventual changes in technique and, consequently, reduced injury rates.

We also recognise that, in order to evaluate GraFeet, we needed to make a choice of a baseline interface. While the state-of-the-art desktop solution used in our study represents a strong baseline, it also poses some challenges. The Runscribe dashboard was most likely designed with the expert user mind. Thus, some advantages of GraFeet revealed in our study may be due to the data filtering applied or the novelty of the form factor. However, comparing GraFeet to a second custom desktop interface would have prevented us from relating our insights to existing artefacts. We recognise that while our design offers certain advantages over the desktop-based dashboard, it also limits some functionalities. Our design emphasises instantaneous metrics and enables users to reflect on how environmental factors (e.g. fatigue, terrain configuration) impacted their gait at different instances of time. This is done at the price of not using time series plots, which may lead to users failing to miss major trends in the data. Further, future research should investigate how to convey running physiology data on screens and compare it with tangible artefacts. For example, GraFeet could be compared with equivalent full-screen visualisations. We hope that GraFeet can inspire other systems which support amateur runners in making sense of their data with the means of augmented running gear (e.g. interactive visualisations on the actual running shoe [11], visualisations of a running shoe on a desktop computer). Future work should study the comparison between such solutions and GraFeet to explore the intricacies of how such solutions might address user needs and support sensemaking.

Finally, we hope that, in future research, we can observe the usage of foot-worn sensors over multiple months or even seasons. We aimed to build a tool that fosters reflection which serves as start for enabling long-term usage and the user learning what metrics indicate that their body is perfectly healthy. A long-term study is required to investigate further if reflection can enable users to detect an oncoming injury or overtraining. Such a study will be able to verify if bodily sensing can become a useful tool for adjusting training routines. Additionally, in order to investigate GraFeet's long-term effect on the users, we would also need to evaluate its utility as a situated artefact. Past work indicates that post-workout reflection can happen primarily at home $[23,40]$ and we would need to verify how GraFeet can fit into the domestic environment.

\section{CONCLUSION}

In this paper, we investigated data-driven post-workout reflection for runners based on body-worn sensors. We designed, implemented and evaluated GraFeet, which visualised foot metrics on a running shoe. Based on data gathered from the Runscribe sensor, GraFeet presented pronation, foot strike type and impact Gs through an LED matrix on the outsole of the shoe. We compared GraFeet with the standard Runscribe dashboard interface in a between-subjects study. We found that GraFeet was perceived as more usable. The users of GraFeet generated more data insights than dashboard users and they were significantly less often confused. Based on our results, we postulate that future sensor data interfaces for sport should manage the number of metrics displayed, explore the post-workout experience, provide reference metrics and communicate background knowledge.

In future work, we hope to conduct a long-term study where users can reflect on their foot mechanics on a daily basis. We envision that future research can extend the capabilities of our prototype by using wearable display technologies. An emerging challenge is also how systems can automatically provide suggestions for improvement based on gait data or support the user in reflecting through data analysis. We hope that this paper will inspire further inquiry into how body-worn sensors can help athletes understand their bodies and eventually avoid injuries.

\section{ACKNOWLEDGMENTS}

This research was financially supported by Utrecht University's Focus Area: Sports and Society and the European Union's Horizon 2020 Programme under ERCEA grant no. 683008 AMPLIFY. We acknowledge the support of the Leibniz ScienceCampus Bremen Digital Public Health (lsc-diph.de), which is jointly funded by the Leibniz Association (W4/2018), the Federal State of Bremen and the Leibniz Institute for Prevention Research and Epidemiology-BIPS.

\section{REFERENCES}

[1] Swamy Ananthanarayan, Nathan Lapinski, Katie Siek, and Michael Eisenberg. 2014. Towards the Crafting of Personal Health Technologies. In Proceedings of the 2014 Conference on Designing Interactive Systems (Vancouver, BC, Canada) (DIS '14). ACM, New York, NY, USA, 587-596. https://doi.org/10.1145/2598510.2598581

[2] Aaron Bangor, Philip T. Kortum, and James T. Miller. 2008. An Empirical Evaluation of the System Usability Scale. International fournal of Human-Computer Interaction 24, 6 (2008), 574-594. https://doi.org/10.1080/10447310802205776

[3] Eric P.S. Baumer. 2015. Reflective Informatics: Conceptual Dimensions for Designing Technologies of Reflection. In Proceedings of the 33rd Annual ACM Conference on Human Factors in Computing Systems (Seoul, Republic of Korea) (CHI '15). ACM, New York, NY, USA, 585-594. https://doi.org/10.1145/2702123.2702234

[4] Eric P.S. Baumer, Vera Khovanskaya, Mark Matthews, Lindsay Reynolds, Victoria Schwanda Sosik, and Geri Gay. 2014. Reviewing Reflection: On the Use of Reflection in Interactive System Design. In Proceedings of the 2014 Conference on Designing Interactive Systems (Vancouver, BC, Canada) (DIS '14). ACM, New York, NY, USA, 93-102. https://doi.org/10.1145/2598510.2598598

[5] Nabil Bin Hannan, Felwah Alqahtani, and Derek Reilly. 2016. JogChalking: Capturing and Visualizing Affective Experience for Recreational Runners. In Proceedings of the 2016 ACM Conference Companion Publication on Designing Interactive Systems (Brisbane, QLD, Australia) (DIS '16 Companion). ACM, New York, NY, USA, 129-132. https://doi.org/10.1145/2908805.2909406

[6] Ann Blandford, Dominic Furniss, and Stephann Makri. 2016. Qualitative HCI Research: Going Behind the Scenes. Morgan \& Claypool Publishers.

[7] Henning Boecker, Till Sprenger, Mary E. Spilker, Gjermund Henriksen, Marcus Koppenhoefer, Klaus J. Wagner, Michael Valet, Achim Berthele, and Thomas R. Tolle. 2008. The Runner's High: Opioidergic Mechanisms in the Human Brain. Cerebral Cortex 18, 11 (2008), 2523-2531. https://doi.org/10.1093/cercor/bhn013

[8] John Brooke. 1996. SUS-A quick and dirty usability scale. Usability evaluation in industry 189, 194 (1996), 4-7.

[9] Hronn Brynjarsdottir, Maria Håkansson, James Pierce, Eric Baumer, Carl DiSalvo, and Phoebe Sengers. 2012. Sustainably Unpersuaded: How Persuasion Narrows Our Vision of Sustainability. In Proceedings of the SIGCHI Conference on Human Factors in Computing Systems (Austin, Texas, USA) (CHI '12). ACM, New York, NY, USA, 947-956. https://doi.org/10.1145/2207676.2208539

[10] Peter R Cavanagh. 1990. Biomechanics of Distance Running. ERIC

[11] Ashley Colley, Paweł W. Woźniak, Francisco Kiss, and Jonna Häkkilä. 2018. Shoe Integrated Displays: A Prototype Sports Shoe Display and Design Space. In Proceedings of the 10th Nordic Conference on Human-Computer Interaction (Oslo, 
Norway) (NordiCHI '18). ACM, New York, NY, USA, 39-46. https://doi.org/10. $1145 / 3240167.3240216$

[12] Franco Curmi, Maria Angela Ferrario, Jen Southern, and Jon Whittle. 2013 HeartLink: Open Broadcast of Live Biometric Data to Social Networks. In CHI '13 Extended Abstracts on Human Factors in Computing Systems (Paris, France) (CHI EA '13). Association for Computing Machinery, New York, NY, USA, 2793-2794. https://doi.org/10.1145/2468356.2479515

[13] Daniel A. Epstein, An Ping, James Fogarty, and Sean A. Munson. 2015. A Lived Informatics Model of Personal Informatics. In Proceedings of the 2015 ACM International Joint Conference on Pervasive and Ubiquitous Computing (Osaka, Japan) (UbiComp '15). ACM, New York, NY, USA, 731-742. https: //doi.org/10.1145/2750858.2804250

[14] Special Eurobarometer. 2014. 412 - Sport and physical activity. European Comission (2014)

[15] Stephen Few. 2006. Information dashboard design. (2006).

[16] Robert Fisher. 1993. Social desirability bias and the validity of indirect questioning 30 (01 1993), 3-15.

[17] Tian Hao, Guoliang Xing, and Gang Zhou. 2015. RunBuddy: A Smartphone System for Running Rhythm Monitoring. In Proceedings of the 2015 ACM International foint Conference on Pervasive and Ubiquitous Computing (Osaka, Japan) (UbiComp '15). ACM, New York, NY, USA, 133-144. https://doi.org/10.1145/2750858.2804293

[18] Mahmoud Hassan, Florian Daiber, Frederik Wiehr, Felix Kosmalla and Antonio Krüger. 2017. FootStriker: An EMS-based Foot Strike Assistant for Running. Proc ACM Interact. Mob. Wearable Ubiquitous Technol. 1, 1, Article 2 (March 2017), 18 pages. https://doi.org/10.1145/3053332

[19] John P Higgins. 2016. Smartphone applications for patients' health and fitness. The American journal of medicine 129, 1 (2016), 11-19.

[20] Kasper Hornbæk et al. 2013. Some whys and hows of experiments in humancomputer interaction. Foundations and Trends ${ }^{\circledR}$ in Human-Computer Interaction 5, 4 (2013), 299-373.

[21] Yvonne Jansen, Pierre Dragicevic, and Jean-Daniel Fekete. 2013. Evaluating the Efficiency of Physical Visualizations. In Proceedings of the SIGCHI Conference on Human Factors in Computing Systems (Paris, France) (CHI '13). ACM, New York, NY, USA, 2593-2602. https://doi.org/10.1145/2470654.2481359

[22] Rohit A. Khot. 2013. Sweat-atoms: Crafting Physical Objects with Everyday Exercise. In CHI '13 Extended Abstracts on Human Factors in Computing Systems (Paris, France) (CHI EA '13). ACM, New York, NY, USA, 2701-2706. https: //doi.org/10.1145/2468356.2479496

[23] Kristina Knaving and PawełWózniak. 2016. TickTockRun: Towards Enhancing Communication in Runner Families. In Proceedings of the 19th ACM Conference on Computer Supported Cooperative Work and Social Computing Companion (San Francisco, California, USA) (CSCW'16 Companion). ACM, New York, NY, USA 309-312. https://doi.org/10.1145/2818052.2869114

[24] Kristina Knaving, Paweł Woźniak, Morten Fjeld, and Staffan Björk. 2015. Flow is Not Enough: Understanding the Needs of Advanced Amateur Runners to Design Motivation Technology. In Proceedings of the 33rd Annual ACM Conference on Human Factors in Computing Systems (Seoul, Republic of Korea) (CHI '15). ACM, New York, NY, USA, 2013-2022. https://doi.org/10.1145/2702123.2702542

[25] Ian Li, Anind Dey, and Jodi Forlizzi. 2010. A Stage-Based Model of Personal Informatics Systems. In Proceedings of the SIGCHI Conference on Human Factors in Computing Systems (Atlanta, Georgia, USA) (CHI '10). Association for Computing Machinery, New York, NY, USA, 557-566. https://doi.org/10.1145/1753326.1753409

[26] Matthew Mauriello, Michael Gubbels, and Jon E. Froehlich. 2014. Social Fabric Fitness: The Design and Evaluation of Wearable E-textile Displays to Support Group Running. In Proceedings of the SIGCHI Conference on Human Factors in Computing Systems (Toronto, Ontario, Canada) (CHI '14). ACM, New York, NY, USA, 2833-2842. https://doi.org/10.1145/2556288.2557299

[27] Richard E Mayer and Roxana Moreno. 2003. Nine ways to reduce cognitive load in multimedia learning. Educational psychologist 38, 1 (2003), 43-52.

[28] David K. McGookin and Stephen A. Brewster. 2013. Investigating and Supporting Undirected Navigation for Runners. In CHI '13 Extended Abstracts on Human Factors in Computing Systems (Paris, France) (CHI EA '13). ACM, New York, NY, USA, 1395-1400. https://doi.org/10.1145/2468356.2468605

[29] Yevgeniy Medynskiy, Svetlana Yarosh, and Elizabeth Mynatt. 2011. Five Strategies for Supporting Healthy Behavior Change. In CHI '11 Extended Abstracts on Human Factors in Computing Systems (Vancouver, BC, Canada) (CHI EA '11). ACM, New York, NY, USA, 1333-1338. https://doi.org/10.1145/1979742.1979770

[30] Thomas S Miller. 2002. Programmed to run. Human Kinetics Publishers.

[31] Florian 'Floyd' Mueller, Shannon O’Brien, and Alex Thorogood. 2007. Jogging over a Distance: Supporting a "Jogging Together" Experience Although Being Apart. In CHI '07 Extended Abstracts on Human Factors in Computing Systems (San Jose, CA, USA) (CHI EA '07). ACM, New York, NY, USA, 1989-1994. https: //doi.org/10.1145/1240866.1240937

[32] Jasmin Niess and Paweł W. Woźniak. 2018. Supporting Meaningful Personal Fitness: The Tracker Goal Evolution Model. In Proceedings of the $2018 \mathrm{CH}$ Conference on Human Factors in Computing Systems (Montreal QC, Canada) (CHI '18). Association for Computing Machinery, New York, NY, USA, 1-12. https://doi.org/10.1145/3173574.3173745
[33] Stina Nylander, Mattias Jacobsson, and Jakob Tholander. 2014. Runright: Realtime Visual and Audio Feedback on Running. In CHI '14 Extended Abstracts on Human Factors in Computing Systems (Toronto, Ontario, Canada) (CHI EA '14). ACM, New York, NY, USA, 583-586. https://doi.org/10.1145/2559206.2574806

[34] Stina Nylander, Alex Kent, and Jakob Tholander. 2014. Swing Sound: Experiencing the Golf Swing Through Sound. In CHI '14 Extended Abstracts on Human Factors in Computing Systems (Toronto, Ontario, Canada) (CHI EA '14). ACM, New York, NY, USA, 443-446. https://doi.org/10.1145/2559206.2574789

[35] Pete Pfitzinger and Scott Douglas. 2009. Advanced Marathoning 2nd Edition. Human Kinetics.

[36] D Poppel, J Koning, AP Verhagen, and GGM Scholten-Peeters. 2016. Risk factors for lower extremity injuries among half marathon and marathon runners of the Lage Landen Marathon Eindhoven 2012: a prospective cohort study in the Netherlands. Scandinavian journal of medicine \& science in sports 26, 2 (2016), 226-234.

[37] Herman Saksono and Andrea G. Parker. 2017. Reflective Informatics Through Family Storytelling: Self-discovering Physical Activity Predictors. In Proceedings of the 2017 CHI Conference on Human Factors in Computing Systems (Denver, Colorado, USA) (CHI '17). ACM, New York, NY, USA, 5232-5244. https://doi. org $/ 10.1145 / 3025453.3025651$

[38] Bruno Tirotti Saragiotto, Tiê Parma Yamato, and Alexandre Dias Lopes. 2014. What Do Recreational Runners Think About Risk Factors for Running Injuries? A Descriptive Study of Their Beliefs and Opinions. Fournal of Orthopaedic \& Sports Physical Therapy 44, 10 (2014), 733-738. https://doi.org/10.2519/jospt.2014.5710 PMID: 25155860.

[39] Purvi Saraiya, Chris North, and Karen Duca. 2005. An insight-based methodology for evaluating bioinformatics visualizations. IEEE transactions on visualization and computer graphics 11, 4 (2005), 443-456.

[40] Kim Sauvé, Steven Houben, Nicolai Marquardt, Saskia Bakker, Bart Hengeveld, Sarah Gallacher, and Yvonne Rogers. 2017. LOOP: A Physical Artifact to Facilitate Seamless Interaction with Personal Data in Everyday Life. In Proceedings of the 2017 ACM Conference Companion Publication on Designing Interactive Systems (Edinburgh, United Kingdom) (DIS '17 Companion). ACM, New York, NY, USA, 285-288. https://doi.org/10.1145/3064857.3079175

[41] Richard Shipway and Immy Holloway. 2010. Running free: Embracing a healthy lifestyle through distance running. Perspectives in public health 130, 6 (2010), 270-276.

[42] Petr Slovák, Christopher Frauenberger, and Geraldine Fitzpatrick. 2017. Reflective Practicum: A Framework of Sensitising Concepts to Design for Transformative Reflection. In Proceedings of the 2017 CHI Conference on Human Factors in Computing Systems (Denver, Colorado, USA) (CHI '17). ACM, New York, NY, USA, 2696-2707. https://doi.org/10.1145/3025453.3025516

[43] Yannick Stephan, Thomas Deroche, Britton W Brewer, Johan Caudroit, and Christine Le Scanff. 2009. Predictors of perceived susceptibility to sport-related injury among competitive runners: The role of previous experience, neuroticism, and passion for running. Applied Psychology 58, 4 (2009), 672-687.

[44] Christina Strohrmann, Holger Harms, Gerhard Tröster, Stefanie Hensler, and Roland Müller. 2011. Out of the Lab and into the Woods: Kinematic Analysis in Running Using Wearable Sensors. In Proceedings of the 13th International Conference on Ubiquitous Computing (Beijing, China) (UbiComp '11). ACM, New York, NY, USA, 119-122. https://doi.org/10.1145/2030112.2030129

[45] Christina Strohrmann, Julia Seiter, Yurima Llorca, and Gerhard Tröster. 2013. Can smartphones help with running technique? Procedia Computer Science 19 (2013), 902-907.

[46] Jakob Tholander and Stina Nylander. 2015. Snot, Sweat, Pain, Mud, and Snow: Performance and Experience in the Use of Sports Watches. In Proceedings of the 33rd Annual ACM Conference on Human Factors in Computing Systems (Seoul, Republic of Korea) (CHI '15). ACM, New York, NY, USA, 2913-2922. https: //doi.org/10.1145/2702123.2702482

[47] Janko Timmermann, Alexander Erlemann, Wilko Heuten, and Susanne Boll. 2014. Supporting Running Groups As a Whole. In Proceedings of the 8th Nordic Conference on Human-Computer Interaction: Fun, Fast, Foundational (Helsinki, Finland) (NordiCHI '14). ACM, New York, NY, USA, 971-974. https://doi.org/10. $1145 / 2639189.2670253$

[48] Wouter Walmink, Alan Chatham, and Florian Mueller. 2013. Lumahelm: An Interactive Helmet. In CHI '13 Extended Abstracts on Human Factors in Computing Systems (Paris, France) (CHI EA '13). ACM, New York, NY, USA, 2847-2848. https://doi.org/10.1145/2468356.2479542

[49] Paweł Woźniak, Kristina Knaving, Staffan Björk, and Morten Fjeld. 2015. RUFUS: Remote Supporter Feedback for Long-Distance Runners. In Proceedings of the 17th International Conference on Human-Computer Interaction with Mobile Devices and Services (Copenhagen, Denmark) (MobileHCI '15). ACM, New York, NY, USA, 115-124. https://doi.org/10.1145/2785830.2785893

[50] Paweł W. Wozniak, Anton Fedosov, Eleonora Mencarini, and Kristina Knaving. 2017. Soil, Rock, and Snow: On Designing for Information Sharing in Outdoor Sports. In Proceedings of the 2017 Conference on Designing Interactive Systems (Edinburgh, United Kingdom) (DIS '17). ACM, New York, NY, USA, 611-623. https://doi.org/10.1145/3064663.3064741 